\title{
Cross-over versus first-order phase transition in holographic gravity-single-dilaton models of QCD thermodynamics
}

\author{
R. Yaresko ${ }^{1,2, a}$, J. Knaute ${ }^{1,2}$, B. Kämpfer ${ }^{1,2}$ \\ ${ }^{1}$ Helmholtz-Zentrum Dresden-Rossendorf, POB 5101 19, 01314 Dresden, Germany \\ ${ }^{2}$ Institut für Theoretische Physik, TU Dresden, 01062 Dresden, Germany
}

Received: 8 April 2015 / Accepted: 10 June 2015 / Published online: 27 June 2015

(C) The Author(s) 2015. This article is published with open access at Springerlink.com

\begin{abstract}
A dilaton potential is adjusted to recently confirmed lattice QCD thermodynamics data in the temperature range $(0.7 \ldots 3.5) T_{c}$ where $T_{c}=155 \mathrm{MeV}$ is the pseudocritical temperature. The employed holographic model is based on a gravity-single-field dilaton dual. We discuss conditions for enforcing (for the pure gluon plasma) or avoiding (for the QCD quark-gluon plasma) a first-order phase transition, but still keeping a softest point (minimum of sound velocity).
\end{abstract}

\section{Introduction}

The celebrated AdS/CFT correspondence [1-3] has sparked a large number of dedicated investigations of strongly coupled systems (cf. [4,5] for recent surveys). A particular field of application is provided by the strong coupling nature of QCD at low momentum/energy scales. While employing $5 \mathrm{~d}$ Einstein gravity in the dual description is strictly justified only in the large $N_{c}$ and large 't Hooft coupling limits of the boundary theory, which ensure suppression of loop and stringy corrections to classical gravitation theory, by such means nevertheless one could study models which are expected to exhibit a behavior resemblant of QCD. The aim is then often to understand, on a qualitative level, phenomena which are hardly accessible in the $4 \mathrm{~d}$ quantum field theory. A prominent example is given by real-time phenomena, e.g. within QCD. Other phenomena, such as the hadron spectrum or the equation of state, are accessible by lattice QCD calculations - but here one would like to understand qualitatively the emerging numerical results by means of transparent models.

Many facets of the QCD equation of state are fairly known by now, both for the physical parameter section and for various limits of parameters (e.g. quark masses, dimen-

\footnotetext{
a e-mail: r.yaresko@hzdr.de
}

sion of the gauge group, flavor number, adjoint representations of quarks etc.). This statement applies only for finitetemperature $(T)$ QCD at zero baryo-chemical potential $(\mu)$. However, in relativistic heavy-ion collisions, the bulk of excited matter has $\mu>0$, as inferred from the analysis of hadron abundancies [6]. The knowledge of the QCD equation of state is, therefore, presently incomplete (in particular beyond the range accessible by the $\mu / T \ll 1$ expansion) and calls urgently for an improvement. In particular, there are several ideas that QCD allows for a critical point in the $T-\mu$ phase diagram where the cross-over turns in a first-order phase transition. Mainly based on universality arguments, a multitude of models have been employed to locate the critical point [7-10], but also more directly QCD anchored approaches, e.g. Dyson-Schwinger equations as integral formulation of QCD, have been used [11]. Parallel to the theoretical attempts, also special experimental searches are conducted, e.g. the beam energy scan at RHIC $[12,13]$.

Coming back to options for modeling a phase diagram similar to QCD with the conjectured critical point, we mention $[14,15]$, where in a holographic model, including gravity, a dilaton field and a U(1) gauge field, the possibility of such a realization has been demonstrated. The set-up of $[14,15]$ is based on a dilation potential, which features qualitatively the equation of state at $\mu=0$, supplemented by a dynamical strength function, which is adjusted to the quark number susceptibility, again at $\mu=0$. While in the infinitely heavy quark mass $\left(m_{q} \rightarrow \infty\right)$ limit of QCD, which becomes then a pure Yang-Mills theory, the equation of state is known since some time [16] and has been confirmed by high-precision lattice QCD simulations [17], the status of QCD with physical quark masses has been settled only very recently. After refinements in the lattice discretization schemes and actions and continuum extrapolations the results of two independent collaborations $[18,19]$ became consistent. 
Given this new situation and having in mind e.g. an application in the spirit of $[14,15]$ to the QCD phase diagram modeling, one should seek for an appropriate dilaton potential, reproducing sufficiently accurately the by now known QCD equation of state at $\mu=0$. This is the aim of the present note. We have hereby the attitude to take the AdS/CFT dictionary literally, i.e. translate, without corrections due to $N_{c}=3$ or finite coupling, the $5 \mathrm{~d}$ Riemann metric into $4 \mathrm{~d}$ energy-momentum tensor components (or correlators) and vice versa.

\section{Adjusting a dilaton potential}

At $\mu=0$, the equation of state, in parametric form, follows from [20] where $p(\infty)=0$ holds if $V^{\prime} / V<2 \sqrt{2 / 3}$ with $V^{\prime} /\left.V\right|_{\phi \rightarrow \infty}$ $\rightarrow$ const, corresponding to a "good" singularity at $\phi=\infty$ [21]. We consider only such cases.

Besides of a proper adjustment of the dilaton potential $V(\phi)$ to the equation of state, the model parameters $G_{5} / L^{3}$ and $L$ must be fitted, too. Since a direct mapping procedure of an input equation of state to the potential is not at our disposal, we use as trial ansatz

$$
\begin{aligned}
& v_{D}(\phi) \equiv \frac{V_{D}^{\prime}}{V_{D}} \\
& = \begin{cases}\frac{-L^{2} M^{2}}{12} \phi+s_{1} \phi^{3} & \text { for } \phi \leq \phi_{m}, \\
\left(t_{1} \tanh \left(t_{2} \phi-t_{3}\right)+t_{4}\right)\left(1-\frac{b_{1}}{\cosh \left(b_{2} \phi-b_{3}\right)^{2}}\right) & \text { for } \phi \geq \phi_{m},\end{cases}
\end{aligned}
$$

(requiring differentiability of $v_{D}$ at $\phi_{m}$ fixes $L^{2} M^{2}$ and $s_{1}$ ) and find

\begin{tabular}{l|l|l|l|l|l|l|l|l|l} 
fit to & $\phi_{m}$ & $t_{1}$ & $t_{2}$ & $t_{3}$ & $t_{4}$ & $b_{1}$ & $b_{2}$ & $b_{3}$ & $G_{5} / L^{3}$ \\
\hline$v_{s}^{2}$ & 0.2163 & 0.6453 & 0.4988 & 0.0845 & 0.0286 & 0.4842 & 2.5020 & 3.9887 & 0.4544 \\
\hline$s / T^{3}$ & 0.2430 & 0.6480 & 0.5023 & 0.0855 & 0.0344 & 0.4844 & 2.6162 & 4.1458 & 0.4586
\end{tabular}

$$
L T\left(\phi_{H}\right)=\frac{V\left(\phi_{H}\right)}{\pi V\left(\phi_{0}\right)} \exp \left(A\left(\phi_{0}\right)+\int_{\phi_{0}}^{\phi_{H}} \mathrm{~d} \phi\left[\frac{1}{4 X}+\frac{2}{3} X\right]\right),
$$

$G_{5} s\left(\phi_{H}\right)=\frac{1}{4} \exp \left(3 A\left(\phi_{0}\right)+\frac{3}{4} \int_{\phi_{0}}^{\phi_{H}} \mathrm{~d} \phi \frac{1}{X}\right)$

for entropy density $s$ and temperature $T$, where the scalar function $X\left(\phi ; \phi_{H}\right)[21]$ is determined by the system (a prime means a derivative w.r.t. $\phi)$

$X^{\prime}=-\left(1+Y-\frac{2}{3} X^{2}\right)\left(1+\frac{3}{4 X} \frac{V^{\prime}}{V}\right)$,

$Y^{\prime}=-\left(1+Y-\frac{2}{3} X^{2}\right) \frac{Y}{X}$,

which is integrated from the horizon $\phi_{H}-\epsilon$ to the boundary $\phi_{0}$ with initial conditions

$$
\begin{aligned}
& X\left(\phi_{H}-\epsilon\right)=-\frac{3}{4} \frac{V^{\prime}\left(\phi_{H}\right)}{V\left(\phi_{H}\right)}+\mathcal{O}\left(\epsilon^{1}\right), \\
& Y\left(\phi_{H}-\epsilon\right)=-\frac{X\left(\phi_{H}-\epsilon\right)}{\epsilon}+\mathcal{O}\left(\epsilon^{0}\right),
\end{aligned}
$$

and $\epsilon \rightarrow 0$. The quantity $A\left(\phi_{0}\right)$ encodes the near-boundary behavior of the model. We assume $L^{2} V(\phi) \approx-12+\frac{L^{2} M^{2}}{2} \phi^{2}$ for $\phi \rightarrow \phi_{0}=0$ which results in $A\left(\phi_{0}\right)=\frac{\log \phi_{0}}{\Delta-4}$, whereby we have set $L \Lambda=1$ [20] and, as usual, $L^{2} M^{2}=\Delta(\Delta-4)$. We consider $2<\Delta<4$.

From $s$ and $T$, the pressure follows as

$$
p\left(\phi_{H}\right)=\int_{\infty}^{\phi_{H}} \mathrm{~d} \tilde{\phi}_{H} \frac{\mathrm{d} T\left(\tilde{\phi}_{H}\right)}{\mathrm{d} \tilde{\phi}_{H}} s\left(\tilde{\phi}_{H}\right)
$$

with $L T_{c}=1.8036$ (fit to $v_{s}^{2}$ from [18]) or $L T_{c}=0.5051$ (fit to $s / T^{3}$ from [18]). This ansatz obeys the ChamblinReall IR behavior $L^{2} V(\phi \rightarrow \infty) \sim \mathrm{e}^{\left(t_{1}+t_{4}\right) \phi}$. The approach belongs to a similar class of holographic models as the model class B in [22]: it has no confinement in the sense of [21] for $t_{1}+t_{4}<\sqrt{2 / 3}$ and no explicit fermionic degrees of freedom. Our ansatz is meant purely to match lattice QCD thermodynamics data in a restricted temperature interval. It is therefore interesting to see in future investigations, e.g. whether the explicit account of quarks has a similar impact on $\zeta / s$ as found in the present setting. To set a scale, we determine $T_{c}$ in the holographic model by the inflection point of $s / T^{3}$ as a function of $T$, and $T_{c}=155 \mathrm{MeV}$ [18] is used in the lattice QCD data [18]. The resulting velocity of sound squared, $v_{s}^{2}=\frac{\mathrm{d} \log T}{\mathrm{~d} \log s}=\frac{\mathrm{d} \log T}{\mathrm{~d} \phi_{H}}\left(\frac{\mathrm{d} \log s}{\mathrm{~d} \phi_{H}}\right)^{-1}$, the scaled entropy density, $s / T^{3}$, the scaled pressure, $p / T^{4}$, and the scaled interaction measure $I / T^{4}=(s T-4 p) / T^{4}$ are exhibited in Fig. 1 together with the lattice QCD data $[18,19]$. The solid blue (dotted red) curves are our optimum fits of $v_{s}^{2}\left(s / T^{3}\right)$ with the parameters of (9). Circles depict the respective quantities at $T_{c}$. One observes that the softest point, i.e. the minimum of $v_{s}^{2}$ as a function of $T / T_{c}$, is slightly below unity (see upper left panel in Fig. 1) and the maximum of the interaction measure is a little bit up-shifted in comparison with the lattice QCD results (see lower right panel in Fig. 1). One observes also some other minor imperfections of our fits, in particular at the lowest temperatures covered by the lattice QCD data, and also at large temperatures for the interaction measure.

In the present setting, the ratio of shear viscosity to entropy density is $\eta / s=1 /(4 \pi)$ [23], as usual for the Hilbert action 

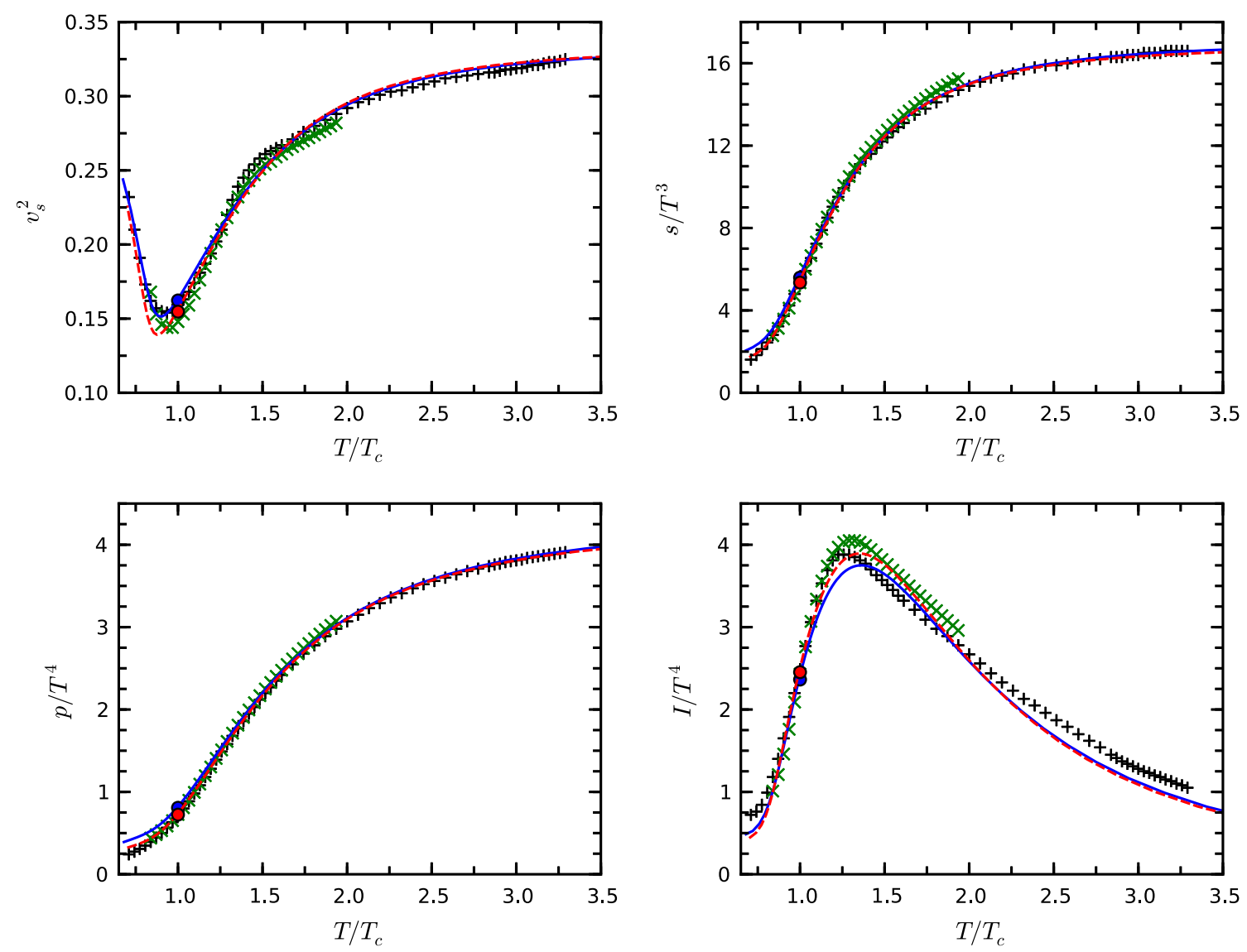

Fig. 1 Velocity of sound squared $v_{s}^{2}$ (upper left panel), scaled entropy density $s / T^{3}$ (upper right panel), scaled pressure $p / T^{4}$ (lower left panel), and scaled interaction measure $I / T^{4}$ (lower right panel) as functions of $T / T_{c}$. Solid blue curves fit to $v_{s}^{2}$ data, dashed red curves fit

to $s / T^{3}$ data, circles position of thermodynamic quantities at $T_{c}$. Lattice QCD data: black plusses from [18], green crosses from [19]; for both sets we use the pseudo-critical temperature $T_{c}=155 \mathrm{MeV}$

on the gravity side $[24,25]$. The ratio of bulk to shear viscosity can be calculated via the Eling-Oz formula [25]

$\left.\frac{\zeta}{\eta}\right|_{\phi_{H}}=\left(\frac{\mathrm{d} \log s}{\mathrm{~d} \phi_{H}}\right)^{-2}=\left(\frac{1}{v_{s}^{2}} \frac{\mathrm{d} \log T}{\mathrm{~d} \phi_{H}}\right)^{-2}$,

or, equivalently $[26,27]$, via the Gubser-Pufu-Rocha formula [24]

$\left.\frac{\zeta}{\eta}\right|_{\phi_{H}}=\frac{V^{\prime}\left(\phi_{H}\right)^{2}}{V\left(\phi_{H}\right)^{2}} \frac{1}{\left|h_{11}\left(\phi_{0}\right)\right|^{2}}$,

where $h_{11}\left(\phi_{0}\right)$ is extracted from solving the perturbation equation

$$
\begin{aligned}
& h_{11}^{\prime \prime}+\frac{1}{X}\left(1+Y-\frac{2}{3} X^{2}\right)\left(2+\frac{9}{4 X} \frac{V^{\prime}}{V}\right) h_{11}^{\prime} \\
& -\frac{Y}{X^{2}}\left(1+Y-\frac{2}{3} X^{2}\right)\left(1+\frac{3}{4 X} \frac{V^{\prime}}{V}\right) h_{11}=0,
\end{aligned}
$$

with initial conditions $h_{11}\left(\phi_{H}-\epsilon\right)=1$ and $h_{11}^{\prime}\left(\phi_{H}-\epsilon\right)=0$ for $\epsilon \rightarrow 0$. The result is exhibited in Fig. 2. Remarkable is the reduction of $\zeta / \eta$ by $50 \%$ at $T_{c}$ in comparison with the $\mathrm{SU}(3)$ gluon plasma (YM) considered in [28]. To understand

this difference, recall the adiabatic approximation of [20]: $X(\phi) \approx-\frac{3}{4} \frac{V^{\prime}(\phi)}{V(\phi)}$. In this approximation, the non-local term in (11) becomes unity, $h_{11}=1$ (since the coefficient of $h_{11}$ in (12) vanishes; see also [29]), and, comparing the values of $V^{\prime} / V \approx 0.6(0.8)$ for the QGP (pure glue; see below) at $T_{c}$ we find the ratio $\left((\zeta / \eta)^{Q G P} /(\zeta / \eta)^{Y M}\right)\left(T_{c}\right) \approx 56 \%$ (cf. also [30,31] for recent holographic calculations of transport coefficients).

On the other hand, for $\zeta / T^{3}$, the situation is reversed: at $T_{c}$, the QGP value is $50 \%$ larger in comparison to the gluon plasma case; the peak of $\zeta / T^{3}$ is located at a larger value $T / T_{c} \approx 1.3$. This difference between $\zeta / \eta$ and $\zeta / T^{3}$ for QGP and for the gluon plasma can be attributed to the different number of degrees of freedom as reflected by the scaled entropy density $s / T^{3}$.

Recent phenomenological investigations [32-35] of the flow harmonics in relativistic heavy-ion collisions emphasize the need to study in more detail the impact of bulk viscosity effects. In this respect, estimates of $\zeta / T^{3}$ in various approaches are of contemporary interest. 

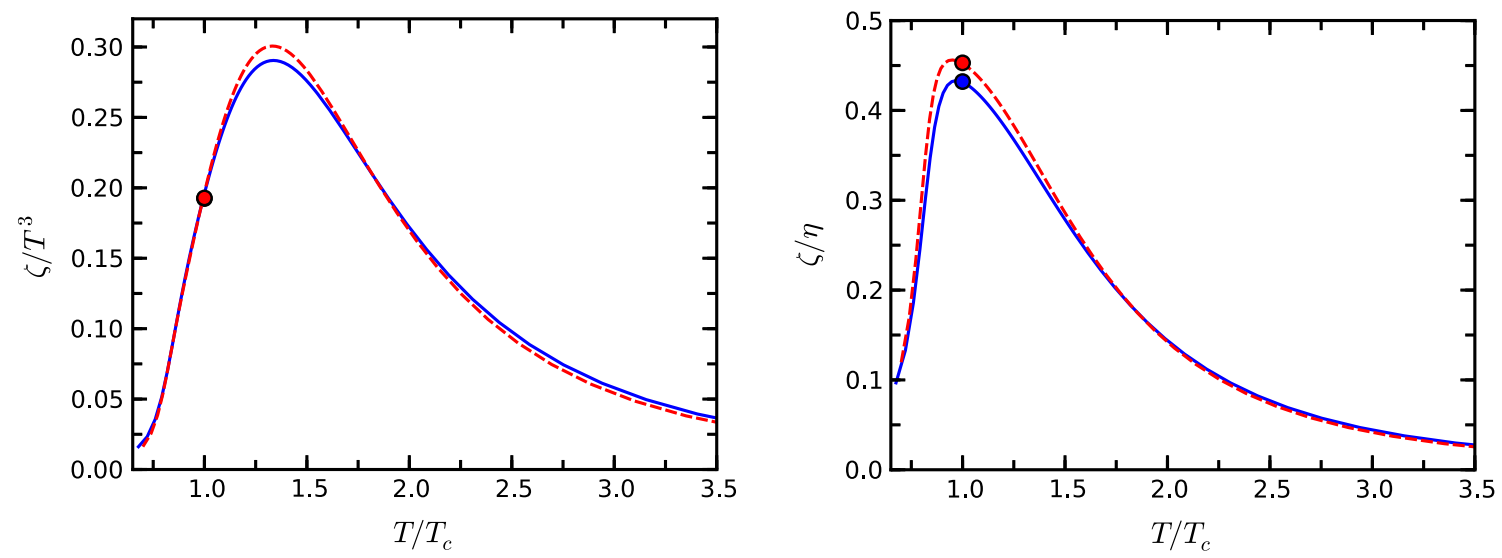

Fig. 2 Scaled bulk viscosity, $\zeta / T^{3}$ (left) and ratio of bulk to shear viscosity, $\zeta / \eta$ (right) as a function of $T / T_{c}$. Line codes as in Fig. 1

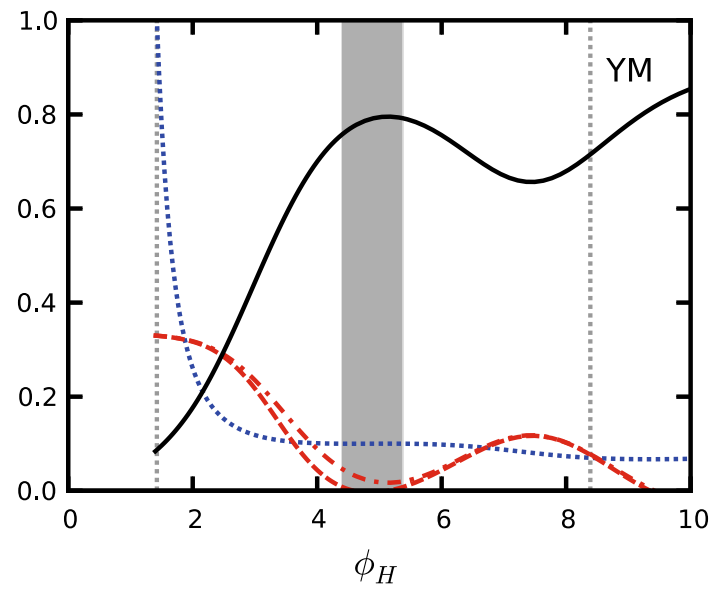

Fig. $3 V^{\prime} / V$ (solid black curves), $v_{s}^{2}$ (dashed red curves, dot-dashed curves are for the adiabatic approximation) and $0.1 T / T_{c}$ (blue dotted curves) as functions of $\phi_{H}$. Left panel for the pure gluon plasma (the

\section{Cross-over vs. first-order phase transition}

Remarkably, the ansatz (8) for the dilaton potential is the same as used in [28] (cf. [36] for a modified variant) to describe the SU(3) gluon plasma, which displays a first-order phase transition. (This is actually not so surprising, as [20] has demonstrated that a two-parameter ansatz for the potential allows either for a cross-over, or a first-order phase transition or a second-order transition, depending on the choice of the parameters. Other examples can be found in [22,37], where one form of the bottom-up ansatz allows for different transition types.) To elucidate the origin of such a difference we exhibit in Fig. 3 a few relevant quantities of both optimized models.

For the pure gluon plasma (left panel) the quantity $V^{\prime} / V$ has a first maximum of about 0.8 at $\phi_{H} \approx 5$.1. In the adiabatic approximation [20] the velocity of sound squared is

$v_{s}^{2} \approx \frac{1}{3}-\frac{1}{2}\left(\frac{V^{\prime}}{V}\right)^{2}+\cdots$

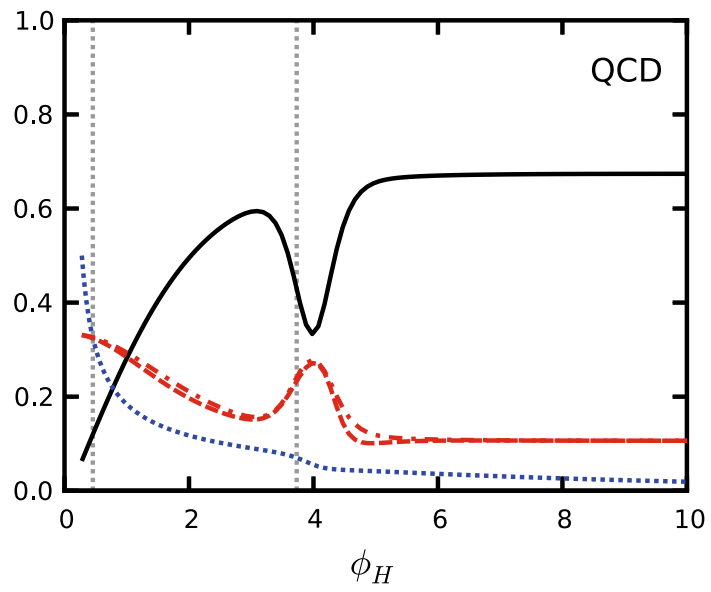

gray band covers the unstable and metastable regions), right panel for QCD quark-gluon plasma (fit to $v_{s}^{2}$ ). Vertical dotted lines bracket the fit range to the lattice data

i.e. a local maximum (minimum) of $V^{\prime} / V$ is related to a local minimum (maximum) of $v_{s}^{2}$. If $V^{\prime} / V$ is sufficiently large, $v_{s}^{2}$ can go to zero. In fact, the adiabatic approximation is quite accurate [compare the red dot-dashed curve (adiabatic approximation) and the dashed curve (exact result) in Fig. 3left panel]. That implies that lifting $V^{\prime} / V$ sufficiently causes a first-order phase transition, here signaled by $v_{s}^{2}=0$. The entropy density $s\left(\phi_{H}\right)$ is a monotonously dropping function, as holds true for the considered examples (and this is assumed to hold in general in the thermodynamically stable phase; see [21]). Hence, $v_{s}^{2}=0$ corresponds to an extremum of $T\left(\phi_{H}\right)$, which is a minimum (maximum) if $\mathrm{d} v_{s}^{2} / \mathrm{d} \phi_{H}<0$ $\left(\mathrm{d} v_{s}^{2} / \mathrm{d} \phi_{H}>0\right)$. Thus, if $V^{\prime} / V$ is adjusted such that $v_{s}^{2}\left(\phi_{H}\right)$ becomes negative in some $\phi_{H}$ interval and then, for larger $\phi_{H}$, rises to become positive again, the local minimum of $T\left(\phi_{H}\right)$ is followed by a local maximum. (These extrema are very shallow in the left panel of Fig. 3 and hardly visible on the used scale.) Such a behavior of $T\left(\phi_{H}\right)$ leads in turn to the usual loop structure of $p(T)$, characteristic of a firstorder phase transition. For the case at hand, the pressure is 
always positive. (In contrast, the IHQCD model [21] has one global minimum of $T\left(\phi_{H}\right)$, which gives rise to the hightemperature branch and the unstable section of $p\left(\phi_{H}\right)$; the low-temperature branch is represented by the line $p=0$ corresponding to the thermal gas.)

Inspection of the same quantities for our fit of the QCD equation of state (see right panel in Fig. 3) reveals $V^{\prime} / V<$ 0.8 everywhere in the considered range of $\phi$ from 0 (UV region) up to 10 (toward the IR region), that is, $v_{s}^{2}>0$ everywhere. Also here, the adiabatic approximation is quite accurate [compare the red dot-dashed curve (adiabatic approximation) and the dashed curve (exact result) in Fig. 3-right panel].

Note that Fig. 3 also unravels some uncomfortable features of the ansatz (8) with parameters adjusted to the lattice YangMills equation of state as in [28]: To catch the shape of thermodynamic quantities in the temperature range $(0.7-10) T_{c}$, the ansatz (8) does not qualify to continue toward the deep IR region, since, e.g., $v_{s}^{2}$ becomes negative for $\phi_{H} \gtrsim 9.5$, signaling the break-down of the ansatz's capabilities. (From the IHQCD viewpoint such a behavior is admissible: the point where $v_{s}^{2}=0$ would signal a Hawking-Page phase transition to the $p=0$ phase, and desirable: the model becomes zero- $T$ confining [21]. In contrast, our ansatz (8) is an ad hoc construction to mimic the Yang-Mills equation of state for $T>0.7 T_{c}$ (up to $10 T_{c}$ ), corresponding to $\phi_{H} \lesssim 8.5$ (down to $\phi_{H} \approx 1.5$ ). It can be supplemented by further terms becoming relevant for $\phi_{H} \gtrsim 8.5$. Thus, it is meaningless to derive from (8) properties of the boundary theory in the IR region.) In contrast, for the QCD parameter adjustment [see (9)], the ansatz (8) seems to be applicable toward the deep IR region.

Upon an integration of $V^{\prime} / V$ the potentials $V(\phi)$ emerge, displayed in Fig. 4. In contrast to $V^{\prime} / V$, the potentials look quite featureless, both in the region where the softest point

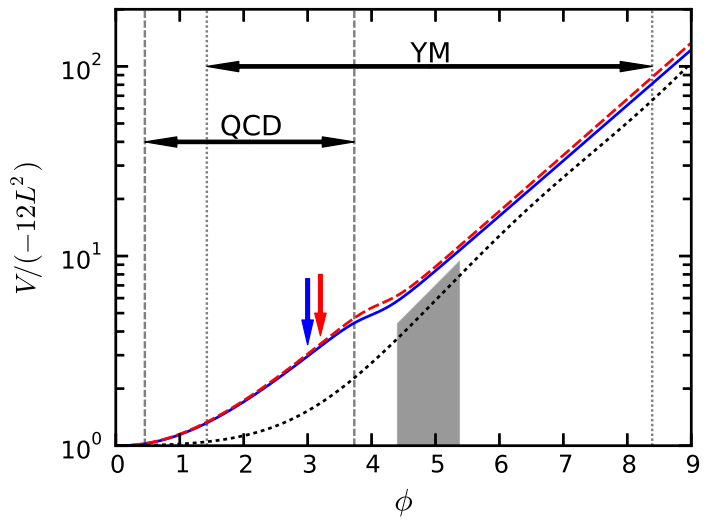

Fig. 4 The dilaton potentials $V(\phi)$ in units of $-12 L^{2}$ for the ansatz (8) with parameter sets from (9) (solid blue or red dashed curve for the fit of $v_{s}^{2}$ or $s / T^{3}$; the arrows point to the location where $v_{s}^{2}$ has the minimum). The potential (8) with parameters adjusted to the Yang-Mills equation of state [28] is exhibited by the dotted black curve (the un/metastable region is depicted by the gray polygon). Vertical dashed (dotted) lines bracket the fit range to QCD [18] (Yang-Mills [17]) lattice data (minimum of $v_{s}^{2}$ ) appears for the QCD equation of state (depicted by the arrows) and in the region of the first-order phase transition for the Yang-Mills equation of state (gray polygon).

\section{Discussion and summary}

In contrast to the IHQCD model, which covers quite a lot of QCD features both for the pure gluon plasma [21,38] and for QCD in the Veneziano limit [39], at finite as well as at zero temperature together with a direct account of the two-loop 't Hooft running coupling, we consider here a simple holographic gravity-single-dilaton model without any explicit a priori scale setting. All parameters are adjusted to finite-temperature lattice QCD thermodynamics in a selected temperature range. We formulate a simple criterion to see already at the dilaton potential (actually its scaled derivative) whether a first-order phase transition can emerge, as for the pure gluon plasma, or a cross-over is encoded, as for QCD at $\mu=0$. While our focus is clearly on features in a limited temperature range at $T \geq T_{c}$, also some section of the low-temperature region can be successfully accommodated in the model, leaving the deep IR region for further studies. We also stress that we do not require a specified behavior of the model outcome in the UV region. Note here that the influence of both asymptotic regimes on the equation of state in the considered temperature interval is fairly small: as shown in [28] the influence of the UV region on dimensionless thermodynamic quantities should not exceed a few percent; for $T>0.7 T_{c}$, the deep IR region contributes to $p$ and $I$ as a small integration constant, while $s, T, v_{s}^{2}$, and the viscosities are independent of it. Hence, although our dilaton potentials ignore QCD features at $T \rightarrow 0$ and $T \rightarrow \infty$, we argue that they qualify for further investigations. For instance, supplemented by a fit of the quark number susceptibility one can repeat the analysis of $[14,15]$ with an up-to-date input to a holographic study of the phase diagram. Even prior to that we note the interesting drop of the ratio $\zeta / \eta$ by $50 \%$ at $T_{c}$ when including quarks.

Another obvious extension of our studies would be the inclusion of a field dual to the chiral condensate $\langle q \bar{q}\rangle$, which is responsible not only for the breaking of conformal invariance in addition to the gluon condensate as expressed by the trace anomaly but, even more importantly, it is in the chiral limit an order parameter of chiral symmetry breaking in QCD. Extensive investigations in this direction, albeit for the Veneziano limit QCD, were performed in [39], where chiral symmetry breaking is realized by tachyon dynamics.

In summary, we present an adjustment of a single-field dilaton potential to recently confirmed lattice QCD thermodynamics data in the temperature range $(0.7-3.5) T_{c}$. A criterion is delivered for ensuring a cross-over at the softest point. 
Acknowledgments The work is supported by BMBF Grant 05P12CRGH1 and European Network HP3-PR1-TURHIC. Inspiring discussions with J. Randrup are gratefully acknowledged.

Open Access This article is distributed under the terms of the Creative Commons Attribution 4.0 International License (http://creativecomm ons.org/licenses/by/4.0/), which permits unrestricted use, distribution, and reproduction in any medium, provided you give appropriate credit to the original author(s) and the source, provide a link to the Creative Commons license, and indicate if changes were made.

Funded by SCOAP ${ }^{3}$.

\section{References}

1. J.M. Maldacena, Adv. Theor. Math. Phys. 2, 231 (1998)

2. S.S. Gubser, I.R. Klebanov, A.M. Polyakov, Phys. Lett. B 428, 105 (1998)

3. E. Witten, Adv. Theor. Math. Phys. 2, 253 (1998)

4. O. DeWolfe, S.S. Gubser, C. Rosen, D. Teaney, Prog. Part. Nucl. Phys. 75, 86 (2014)

5. N. Brambilla et al., Eur. Phys. J. C 74, 2981 (2014)

6. A. Andronic, P. Braun-Munzinger, J. Stachel, Nucl. Phys. A 772, 167 (2006)

7. B. Friman, C. Hohne, J. Knoll, S. Leupold, J. Randrup, R. Rapp, P. Senger (eds.), Lect. Notes Phys. 814, 1 (2011)

8. M.A. Stephanov, Acta Phys. Polon. B 35, 2939 (2004); Prog. Theor. Phys. Suppl. 153, 139 (2004)

9. M.A. Stephanov, Int. J. Mod. Phys. A 20, 4387 (2005)

10. M.A. Stephanov, PoS LAT2006, 024 (2006)

11. C.S. Fischer, J. Luecker, C.A. Welzbacher, Nucl. Phys. A 931, 774 (2014)

12. G. Odyniec, PoS CPOD, 043 (2013)

13. L. Kumar, Mod. Phys. Lett. A 28, 1330033 (2013)

14. O. DeWolfe, S.S. Gubser, C. Rosen, Phys. Rev. D 83, 086005 (2011)
15. O. DeWolfe, S.S. Gubser, C. Rosen, Phys. Rev. D 84, 126014 (2011)

16. G. Boyd, J. Engels, F. Karsch, E. Laermann, C. Legeland, M. Lutgemeier, B. Petersson, Nucl. Phys. B 469, 419 (1996)

17. S. Borsanyi, G. Endrodi, Z. Fodor, S.D. Katz, K.K. Szabo, JHEP 07, 056 (2012)

18. S. Borsanyi et al., Phys. Lett. B 730, 99 (2014)

19. A. Bazavov et al., Phys. Rev. D 90, 094503 (2014)

20. S.S. Gubser, A. Nellore, Phys. Rev. D 78, 086007 (2008)

21. U. Gursoy, E. Kiritsis, L. Mazzanti, F. Nitti, JHEP 05, 033 (2009)

22. S.I. Finazzo, J. Noronha, Phys. Rev. D 90, 115028 (2014)

23. P. Kovtun, D.T. Son, A.O. Starinets, Phys. Rev. Lett. 94, 111601 (2005)

24. S.S. Gubser, S.S. Pufu, F.D. Rocha, JHEP 08, 085 (2008)

25. C. Eling, Y. Oz, JHEP 06, 007 (2011)

26. A. Buchel, JHEP 1105, 065 (2011)

27. A. Buchel, U. Gursoy, E. Kiritsis, JHEP 09, 095 (2011)

28. R. Yaresko, B. Kampfer, arXiv:1306.0214v3

29. U. Gursoy, E. Kiritsis, G. Michalogiorgakis, F. Nitti, JHEP 12, 056 (2009)

30. D. Li, S. He, M. Huang, JHEP 06, 046 (2015)

31. S.I. Finazzo, R. Rougemont, H. Marrochio, J. Noronha, JHEP 02, 051 (2015)

32. K. Dusling, T. Schäfer, Phys. Rev. C 85, 044909 (2012)

33. Y. Hatta, J. Noronha, G. Torrieri, B.-W. Xiao, Phys. Rev. D 90, $074026(2014)$

34. J. Noronha-Hostler, J. Noronha, F. Grassi, Phys. Rev. C 90, 034907 (2014)

35. F.G. Gardim, J. Noronha-Hostler, M. Luzum, F. Grassi, Phys. Rev. C 91, 034902 (2015)

36. R. Yaresko, B. Kampfer, Phys. Lett. B 747, 36 (2015)

37. R.A. Janik, G. Plewa, H. Soltanpanahi, M. Spalinski, arXiv: 1503.07149

38. U. Gursoy, E. Kiritsis, L. Mazzanti, G. Michalogiorgakis, F. Nitti, Lect. Notes Phys. 828, 79 (2011)

39. T. Alho, M. Järvinen, K. Kajantie, E. Kiritsis, C. Rosen, K. Tuominen, JHEP 04, 124 (2014) 